\title{
Análisis de la interdisciplinariedad en la ingeniería química universitaria española
}

\author{
María Pilar Lagar-Barbosa*, María Isabel Escalona-Fernández**, Antonio Pulgarín* \\ *Departamento de Información y Comunicación, Facultad de Ciencias de la Documentación y la Comunicación, \\ Universidad de Extremadura. Badajoz. Correo-e: mplagar@unex.es; pulgarin@unex.es \\ **Servicio de Bibliotecas, Archivo y Documentación, Universidad de Extremadura. Cáceres. Correo-e: escalona@unex.es
}

Recibido: 10-12-2012; 2a version: 21-02-2013; Aceptado: 15-04-2013.

Cómo citar este artículo/Citation: Lagar-Barbosa, M. P.; Escalona-Fernández, M. I.; Pulgarín, A. (2014). Análisis de la interdisciplinariedad en la ingeniería química universitaria española. Revista Española de Documentación Científica, $37(1)$ :e029. doi: http://dx.doi.org/10.3989/redc.2014.1.1048

Resumen: El presente trabajo aborda el estudio de la interdisciplinariedad en el área de ingeniería química (IQ). El análisis incluye una descripción detallada de la ocurrencia de documentos y de revistas en cada categoría del Journal Citation Reports (JCR), con el fin de comprender la estructura de conocimiento de las investigaciones en IQ, observar sus patrones y las tendencias asociadas. Mediante la utilización de los métodos bibliométricos se analiza la multiasignación de artículos y/o de revistas a las distintas categorías del JCR. El total de revistas (598) están distribuidas entre 115 categorías. El $54 \%$ de las revistas (324) y el $56 \%$ de los documentos estarían asignados a más de una categoría. Para mapear los datos correspondientes al análisis de coocurrencia de las distintas categorías se utilizó la técnica de Visualización de Similaridades (VOS). Con la coocurrencia de las distintas categorías se construyó una gráfica bidimensional de las proximidades entre ellas para formar distintos clusters. El resultado fueron 13 clusters o grupos. Destacan como categorías más centradas y más relacionadas "Engineering, Chemical", "Chemistry, Physical" y "Biotechnology \& Applied Microbiology". No se ha observado relación entre el índice de coautoría y la multiasignación en categorías. Las 14 categorías seleccionadas como las más productivas presentan, en el año 2006, un 39,7\% de revistas en el $1^{\text {er }}$ cuartil del JCR.

Palabras clave: Interdisciplinariedad; multiasignación; ingeniería química; categorías del Journal Citation Reports; visualización de similaridades.

\section{Analysis of Interdisciplinarity in Chemical Engineering in Spanish Universities}

Abstract: An analysis is made of interdisciplinarity in the chemical engineering (CE) field. It includes a detailed description of the occurrence of papers and journals in each of the Journal Citation Reports (JCR) categories in order to gain insight into the structure of knowledge in CE research, its patterns and associated trends. Bibliometric methods are used to analyse the multi-assignment of papers and/or journals to the various JCR categories. The total of journals (598) is distributed among 115 categories, with $54 \%$ of them (324) and $56 \%$ of the documents assigned to more than one category. The results of a co-occurrence analysis of the data corresponding to the different categories are mapped using the Visualization of Similarities (VOS) technique. The co-occurrences are plotted on a two-dimensional representation revealing the distinct clusters formed by their proximities. The result was 13 clusters. The categories that stand out as most central and most interconnected were "Engineering, Chemical", "Chemistry, Physical", and "Biotechnology \& Applied Microbiology". No relationship was found between the co-authorship index and multi-assignment into categories. The 14 categories selected as the most productive represented, in $2006,39.7 \%$ of the journals in the JCR's 1 st quartile.

Keywords: Interdisciplinarity; multi-assignment; chemical engineering; Journal Citation Reports categories; visualization of similarities.

Copyright: (c) 2014 CSIC. Este es un artículo de acceso abierto distribuido bajo los términos de la licencia Creative Commons Attribution-Non Commercial (by-nc) Spain 3.0. 


\section{INTRODUCCIÓN}

Se dice que un área de conocimiento es más interdisciplinar o interdisciplinaria que otra cuando ésta engloba o supone la colaboración de más disciplinas que aquélla. La interdisciplinariedad es la puesta en común de la intersección de saberes.

Por el contrario, cuando empleamos el término multidisciplinario nos estamos refiriendo a que es común a varias disciplinas.

No cabe duda de que la investigación actual sea más interdisciplinaria que la de ayer y que lo será más a medida que los experimentos científicos se hacen más complejos y necesitan de más especialistas para resolver los problemas. La interdisciplinariedad está cada vez más presente en la investigación. Sin embargo, no se produce por igual en todas las disciplinas científicas.

Por otro lado, los gobiernos, a través de su política científica, conscientes de que el progreso científico tiene que ver con la colaboración científica, han propuesto medidas con objeto de fomentar la colaboración interdisciplinar.

La ingeniería química (IQ) es un campo que está sufriendo cambios significativos en los últimos tiempos. La investigación interdisciplinar del área se ha incrementado notablemente debido a la complejidad de su actividad, que la ha hecho mucho más diversificada y por ende más necesitada del conocimiento de otras disciplinas.

Diversos métodos bibliométricos han sido descritos en anteriores trabajos que abordan el estudio sobre la interdisciplinariedad. Por ejemplo Porter y Chubin (1985) y Cronin y Pearson (1990), trabajan sobre citas; Tijssen (1992), sobre las palabras asociadas; Katz y Hicks (1995), con las revistas interdisciplinares; Sanz y otros (2002) analizan distintos aspectos de la interdisciplinariedad de los investigadores puertorriqueños en ciencias químicas; Morillo y otros (2003) estudian la multiasignación de las revistas en las distintas categorías del Journal Citation Reports (Thomson Reuters). Recientemente, Pino-Díaz y otros (2011) han llevado a cabo un análisis estratégico y dinámico de la Red Española de Investigación sobre Áreas Protegidas y Chang y Huang (2012) han investigado los cambios de la interdisciplinariedad en el área de ciencia de la información, utilizando para ello tres métodos bibliométricos: la citación, el emparejamiento bibliográfico y el análisis de la coautoría. Una revisión de los principales métodos analíticos con algunas aplicaciones nos la proporciona la revisión de Bordons y otros (2004).

Con estos métodos se puede estudiar, por ejemplo, la migración de científicos entre campos (Le Pair, 1980; Hargens, 1986; Urata, 1990), la tipología de disciplinas de acuerdo con el grado de interdisciplinariedad que presentan (Rinia y otros, 2002; Morillo y otros, 2003), la colaboración científica, revelando la interdisciplinariedad de estos se- gún su papel en una publicación (Bordons y otros, 1999; Schoepflin y Glänzel, 2001), el análisis de la multiasignación de artículos o de revistas a las distintas categorías del Journal Citation Reports (Morillo, 2001; Morillo y otros, 2003), la relación entre la interdisciplinariedad y otras variables, como por ejemplo la colaboración (Qin y otros, 1997; Carayol y Thuc Uyen, 2005).

Con objeto de mapear los datos resultantes del análisis de coocurrencia de las distintas categorías, existen técnicas como el Escalamiento Multidimensional (MDS) (Izenman, 2008) o la Visualización de Similaridades (VOS) (Van Eck y Waltman, 2007, 2010). Esta última permite visualizar similitudes entre categorías en un espacio bidimensional. En el presente estudio, VOS utiliza las categorías del JCR como objetos de análisis. La coocurrencia de estas categorías formará una gráfica bidimensional de las proximidades entre ellas. Esto permitirá visualizar la distancia o proximidad entre las categorías, agrupando las de similares características para formar distintos clusters o agrupamientos. Esta técnica la han utilizado autores como Lu y Wolfram (2010) y Tijssen (2010).

El propósito del presente trabajo es analizar el conjunto de disciplinas relacionadas con la IQ en la universidad española, entre 2000 y 2006 . Se pretende mostrar el conjunto de categorías que están relacionadas con el área de Ingeniería Química, observar la estructura del entramado de materias que coadyuvan al desarrollo de la IQ y a la solución de los problemas que se le plantean, observar sus patrones y las tendencias asociadas.

\section{METODOLOGÍA}

Para recuperar la información sobre la IQ de las universidades públicas españolas se procedió del siguiente modo:

- En primer lugar se buscó toda la información concerniente a universidades, departamentos, áreas de conocimiento e investigadores relacionados con la IQ, con el fin de recuperar los registros pertinentes de IQ. Los departamentos universitarios pueden estar constituidos por una o varias áreas y, a su vez, el nombre del área de IQ puede formar parte del departamento o no.

- Teniendo en cuenta lo anterior, se realizaron una serie de búsquedas retrospectivas, en las bases de datos de WoS, introduciendo el nombre del departamento: términos truncados en español, lenguas cooficiales y en inglés.

- Una vez recuperados los registros se procedió a expurgar aquellos que correspondían a investigadores de otras áreas.

- La ecuación de búsqueda tipo, por ejemplo para la Universidad del País Vasco, que cuenta con tres departamentos con área de IQ (Ingeniería Química y Medio Ambiente; Ingeniería Química; 
y Química Aplicada) sería como sigue:

1. $A D=((" i n g *$ quim $O R$ "chem* eng" OR "ing kim*" OR "environ* chem.* eng*" OR "chem.* environ* eng*" OR "quim* apl*" OR "kim* apli*" OR "appl* chem*" OR "chem.* appl*") SAME spain)

2. $A D=((" u n i v$ país vasco" $O R$ "univ basque country" OR UPV OR "Euskal Herico univertsitatea") SAME spain)

3. \#1 AND \#2

EI JCR ofrece una clasificación por categorías (Subject Category Listing) de las revistas indizadas en WoK (Thomson Reuters). Una revista puede estar adscrita a una única categoría o a varias.

En el caso de la producción científica del área de la IQ española analizada (2000-2006), el número de categorías en las que aparecen adscritas las revistas donde se publicaron los artículos fue de 115. Son categorías de temáticas muy variadas. Entre estas, 20 aparecen una sola vez.

La asignación de revistas a más de una disciplina permite establecer relaciones entre ellas, y por tanto, entre los documentos. La asignación de revistas a las categorías temáticas se asocia a la interdisciplinariedad, considerando que las multiasignadas deben ser más interdisciplinares que las asignadas a una única disciplina.

En el estudio, se observa la interdisciplinariedad a través de la asignación de las revistas en las que publicaron los investigadores del área de IQ universitaria española en categorías del JCR (Lagar Barbosa, 2012).

De acuerdo con este criterio, se han calculado los porcentajes de revistas multiasignadas por categoría y se han estudiado las relaciones entre las distintas categorías utilizando la técnica de mapeo por Visualización de Similaridades (Van Eck y Waltman, 2007).
Se muestran las categorías del JCR más productivas, así como el índice de coautoría, para esas categorías, y el número de revistas en el primer cuartil para el año 2006. Igualmente, se muestra la distribución de multiasignación de revistas en las distintas categorías del JCR.

Por último se puede observar el mapa de Visualización de Similaridades, donde se localizan las categorías en el espacio y se ofrece información relacionada con las distintas categorías.

Para probar el contraste estadístico así como para llevar a cabo la correlación entre las distintas variables fue utilizado el paquete estadístico SPSS ver. 19.

\section{RESULTADOS}

El estudio incluye una descripción general de la productividad científica de las disciplinas relacionadas con la IQ en la universidad española a nivel global (2000-2006). La producción total fue de 4.648 documentos, distribuidos en 598 revistas (Lagar-Barbosa, 2012). Igualmente, el estudio analiza las ocurrencias de revistas y documentos en cada categoría del JCR. Para comparar la distribución de fuentes entre las categorías con las de sus referencias bibliográficas, se seleccionaron las correspondientes al año 2006. Los 822 documentos publicados en 2006 generaron un total de 22.969 referencias bibliográficas.

\subsection{Descripción general de la productividad de las categorías del JCR}

La fig. 1 muestra la relación entre revistas y documentos distribuidos entre las 115 categorías del JCR analizadas. Se representa el acumulado de revistas (1.075) frente al acumulado de ocurrencias de documentos (8.426).

Figura 1. Relación entre revistas y documentos

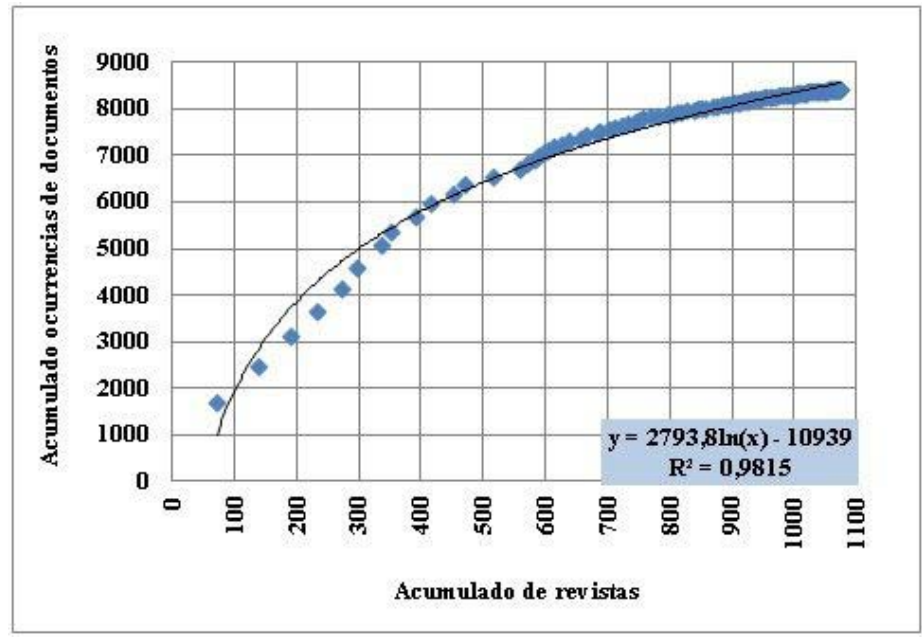


La tabla I muestra las 14 Categorías del JCR más productivas de entre las 115 resultantes del estudio. Las 14 categorías alcanzan el $52 \%$ del total de revistas (559) y cerca del $80 \%$ de los documentos (6.722). Además, se observan los índices de coautoría para esas categorías (4,28 autores/artículo, de media), el porcentaje de multiasignación de cada categoría, el número de revistas situadas en el primer cuartil (Q1) para los documentos publicados en 2006 y el porcentaje de colaboración internacional de cada una de ellas.

El $6,7 \%$ del total de las revistas y más del $20 \%$ del total de documentos están encuadrados en la categoría "Engineering, Chemical", le siguen "Chemistry, Physical", con el 6\%, de revistas y un $9 \%$ de los documentos, "Environmental Sciences", con el $4,9 \%$ y $7,3 \%$ respectivamente, y Biotechnology \& Applied Microbiology, con el $4 \%$ y $6,7 \%$.

Las categorías "Engineering, Environmental" y "Chemistry, Applied" presentan una multiasignación del $100 \%$ (todas las revistas están asignadas a más de una categoría), les siguen "Energy \& Fuels", con más del 94\% y "Water Resources", con el $86 \%$. "Engineering, Chemical" tiene un porcentaje de multiasignación del 70,8\%. De las 14 categorías más productivas, la que cuenta con menor multiasignación es "Chemistry, Multidisciplinary", con un $43,6 \%$.
La visibilidad en cada categoría se puede constatar observando el porcentaje de revistas contenidas en el $1^{\text {er }}$ cuartil (año 2006). La tabla I presenta unos porcentajes de revistas en el Q1 variables, desde un 29\% en "Engineering, Environmental", hasta más del 52\% en "Energy \& Fuels". El 39,7\% de las revistas que integran las 14 categorías seleccionadas estarían situadas en el Q1 de alguna categoría.

Con respecto a la colaboración internacional, las 14 categorías en conjunto, superan el $76 \%$ del total de la colaboración internacional. Las categorías que mayor colaboración presentan son las tres primeras de la tabla I, "Engineering, Chemical", con casi un 17\%, "Chemistry, Physical", con 9,95\% y "Environmental Sciences", con el 7,32\%, le sigue "Polymer Science", con un 6,89\%.

En la tabla II se observan las categorías en las que están encuadradas las fuentes de las referencias correspondientes a 2006, el número de artículos publicados en cada categoría, el porcentaje respecto al total, así como la posición que ocupa la categoría según las categorías a las que pertenecen las revistas donde se publicaron los artículos que constituyen el material de estudio. Las 13 primeras categorías son idénticas a las de la tabla I, aunque el orden varía. La categoría de "Engineering, Chemical" ocupa el primer lugar, con 18

Tabla I. Distribución de revistas entre las categorías más productivas del JCR

\begin{tabular}{|c|c|c|c|c|c|c|}
\hline Categoría del JCR & $\begin{array}{l}\text { No de } \\
\text { revistas }\end{array}$ & $\begin{array}{c}\text { Revistas 1 } \\
\text { cuartil }(\%) \\
(2006)\end{array}$ & $\begin{array}{l}\text { Ocurrencias } \\
\text { documentos }\end{array}$ & $\begin{array}{l}\text { Índice de } \\
\text { coautoría }\end{array}$ & $\begin{array}{l}\text { \% Multi- } \\
\text { asignación }\end{array}$ & $\begin{array}{c}\% \\
\text { Colaboración } \\
\text { internacional }\end{array}$ \\
\hline Engineering, Chemical & 72 & $29(40,2)$ & 1717 & 4,09 & 70,83 & 16,99 \\
\hline Chemistry, Physical & 65 & $22(33,8)$ & 774 & 4,35 & 60,00 & 9,95 \\
\hline Environmental Sciences & 53 & $19(35,8)$ & 619 & 4,32 & 77,36 & 7,32 \\
\hline $\begin{array}{l}\text { Biotechnology \& Applied } \\
\text { Microbiology }\end{array}$ & 43 & $14(32,6)$ & 562 & 4,32 & 69,77 & 5,92 \\
\hline Chemistry, Multidisciplinary & 39 & $15(38,5)$ & 465 & 3,95 & 43,59 & 4,41 \\
\hline Engineering, Environmental & 24 & $7(29,2)$ & 465 & 4,30 & 100,00 & 5,59 \\
\hline Polymer Science & 40 & $17(42,5)$ & 464 & 4,28 & 47,50 & 6,89 \\
\hline Water Resources & 15 & $6(40,0)$ & 320 & 4,35 & 86,67 & 4,95 \\
\hline Food Science \& Technology & 39 & $15(38,5)$ & 298 & 4,04 & 61,54 & 2,37 \\
\hline Chemistry, Applied & 25 & $12(48,0)$ & 285 & 4,40 & 100,00 & 2,74 \\
\hline Chemistry, Analytical & 36 & $15(41,7)$ & 224 & 4,33 & 75,00 & 2,42 \\
\hline Energy \& Fuels & 19 & $10(52,6)$ & 195 & 4,15 & 94,74 & 1,56 \\
\hline Biochemistry \& Molecular Biology & 45 & $20(44,4)$ & 178 & 4,48 & 84,44 & 3,28 \\
\hline $\begin{array}{l}\text { Materials Science, } \\
\text { Multidisciplinary }\end{array}$ & 44 & $21(47,7)$ & 156 & 4,55 & 81,82 & 2,21 \\
\hline Total & 559 & $222(39,7)$ & 6722 & 4,28 & & 76,6 \\
\hline
\end{tabular}


revistas $(20,7 \%)$ y 3.722 documentos $(23,94 \%)$, seguida de "Chemistry, Physical" con 10 revistas $(11,5 \%)$ y 1.704 documentos $(10,96 \%)$ y de "Biotechnology \& Applied Microbiology", con 9 revistas y 1.419 documentos.

\subsection{Multiasignación de revistas y de docu- mentos en categorías del JCR}

La tabla III contiene los datos correspondientes a la multiasignación de revistas, los documentos publicados por éstas, las citas que recibieron esos documentos en 2008 (citas'08), las citas que recibieron en 2011 (citas'11) y el índice de coautoría para cada tipo de multiasignación.
El $54 \%$ de las revistas (324) y el $56 \%$ de los documentos estarían multiasignadas, asignadas a más de una categoría.

El total de revistas (598) están distribuidas entre 115 categorías. Las revistas aparecen multiasignadas entre una y seis categorías. Dos de estas están multiasignadas a seis categorías, mientras que 274 revistas estarían asignadas a una sola categoría $(45,82 \%)$.

No se apreciaron diferencias significativas entre los índices de coautoría de las distintas categorías asignadas, tras ser contrastado con el test no paramétrico de Kruskal-Vallis $\left(\chi^{2}=5 ; p=0,416\right)$.

Tabla II. Distribución de las fuentes correspondientes a las referencias de 2006, según las categorías del JCR

\begin{tabular}{|c|c|c|c|c|c|}
\hline Categoría del JCR & $\begin{array}{l}\text { No de } \\
\text { revistas }\end{array}$ & $\%$ & $\begin{array}{l}\text { Número de } \\
\text { documentos }\end{array}$ & $\%$ & $\begin{array}{c}\text { Posición en } \\
\text { publicaciones }\end{array}$ \\
\hline Engineering, Chemical & 18 & 20,70 & 3722 & 23,94 & 1 \\
\hline Chemistry, Physical & 10 & 11,50 & 1704 & 10,96 & 2 \\
\hline Biotechnology \& Applied Microbiology & 9 & 10,34 & 1419 & 9,13 & 4 \\
\hline Environmental Sciences & 6 & 6,90 & 1397 & 8,99 & 3 \\
\hline Polymer Science & 6 & 6,90 & 1131 & 7,28 & 7 \\
\hline Engineering, Environmental & 5 & 5,75 & 1263 & 8,13 & 6 \\
\hline Chemistry, Analytical & 4 & 4,60 & 387 & 2,49 & 11 \\
\hline Water Resources & 3 & 3,44 & 842 & 5,42 & 8 \\
\hline Chemistry, Multidisciplinary & 3 & 3,44 & 652 & 4,19 & 5 \\
\hline Chemistry, Applied & 3 & 3,44 & 564 & 3,63 & 10 \\
\hline Food Science \& Technology & 3 & 3,44 & 425 & 2,73 & 9 \\
\hline Energy \& Fuels & 2 & 2,30 & 347 & 2,23 & 12 \\
\hline Materials Science, Multidisciplinary & 2 & 2,30 & 212 & 1,36 & 14 \\
\hline Electrochemistry & 2 & 2,30 & 176 & 1,13 & 24 \\
\hline Agricultural Engineering & 1 & 1,15 & 121 & 0,78 & 19 \\
\hline Agricultural Multidisciplinary & 1 & 1,15 & 205 & 1,32 & 26 \\
\hline Biochemistry \& Molecular Biology & 1 & 1,15 & 148 & 0,95 & 13 \\
\hline Microbiology & 1 & 1,15 & 147 & 0,95 & 45 \\
\hline Biochemical Research Methods & 1 & 1,15 & 109 & 0,70 & 23 \\
\hline Thermodynamics & 1 & 1,15 & 105 & 0,68 & 15 \\
\hline Multidisciplinary Sciences & 1 & 1,15 & 103 & 0,66 & 77 \\
\hline Engineering, Civil & 1 & 1,15 & 97 & 0,62 & 17 \\
\hline Physics, Atomic, Molecular \& Chemical & 1 & 1,15 & 90 & 0,58 & 21 \\
\hline Computer Science, Interdisciplinary Applications & 1 & 1,15 & 89 & 0,57 & 30 \\
\hline Metallurgy \&Metallurgical Engineering & 1 & 1,15 & 89 & 0,57 & 20 \\
\hline Total & 87 & 100 & 15.644 & 100 & \\
\hline
\end{tabular}


Entre las citas'08 y las citas' 11 , recibidas por las revistas asignadas a una o más categorías, existe una fuerte correlación ( $r=0,998$ ) (fig. 2).

Las diferencias de medias entre las citas recibidas por las revistas asignadas a una sola categoría $\mathrm{y}$ las multiasignadas no fueron significativas ( $\mathrm{t}=$ 1,$872 ; \mathrm{p}=0,135$, para citas'08 y $\mathrm{t}=1,693 ; \mathrm{p}=$ 0,156, para citas'11).

\subsection{Estructura del área de ingeniería química}

En las figs. 3 y 4 se presentan los mapas realizados con la técnica de Visualización de Similaridades - VOS (Van Eck y Waltman, 2007). La localización de las categorías en el espacio es una representa- ción de similaridad (número de veces que un par de categorías aparecen juntas). El mapa de la fig. 3 ofrece información relacionada con categorías como: "Engineering, Chemical", "Chemistry, Physical" y "Energy \& Fuels"; además de información sobre las relaciones entre los distintos clusters.

De las 115 categorías del análisis, 12 no están conectadas entre sí o sus conexiones son muy débiles. Estas categorías se han omitido a la hora de representar la interdisciplinariedad. Las 103 categorías restantes están conectadas entre sí y distribuidas en 13 clusters (fig. 3). El color de la categoría indica el grupo o cluster al que ha sido asignada y su tamaño muestra la frecuencia con la que esa categoría aparece en el estudio.

Tabla III. Multiasignación de revistas, documentos, citas'08 y citas'11 a categorías del JCR

\begin{tabular}{lccccccccc}
\hline $\begin{array}{l}\text { Categorías } \\
\text { asignadas }\end{array}$ & $\begin{array}{l}\text { Número } \\
\text { revistas }\end{array}$ & $\%$ & $\begin{array}{c}\text { Número } \\
\text { documentos }\end{array}$ & $\%$ & $\begin{array}{c}\text { Citas } \\
\mathbf{2 0 0 8}\end{array}$ & \% & $\begin{array}{c}\text { Citas } \\
\mathbf{2 0 1 1}\end{array}$ & $\begin{array}{c}\text { Índice de } \\
\text { coautoría }\end{array}$ \\
\hline Una & 274 & 45,82 & 2045 & 44,00 & 12520 & 42,03 & 26338 & 40,40 & 4,19 \\
\hline Dos & 212 & 35,45 & 1542 & 33,18 & 8659 & 29,07 & 19022 & 29,18 & 4,17 \\
\hline Tres & 80 & 13,38 & 960 & 20,65 & 7908 & 26,55 & 18425 & 28,26 & 4,35 \\
\hline Cuatro & 25 & 4,18 & 90 & 1,94 & 570 & 1,91 & 1169 & 1,79 & 4,81 \\
\hline Cinco & 5 & 0,84 & 9 & 0,19 & 131 & 0,44 & 224 & 0,35 \\
\hline Seis & 2 & 0,33 & 2 & 0,04 & 1 & 0,00 & 14 & 0,02 \\
\hline
\end{tabular}

Figura 2. Citas'08 y citas' 11 según categorías del JCR

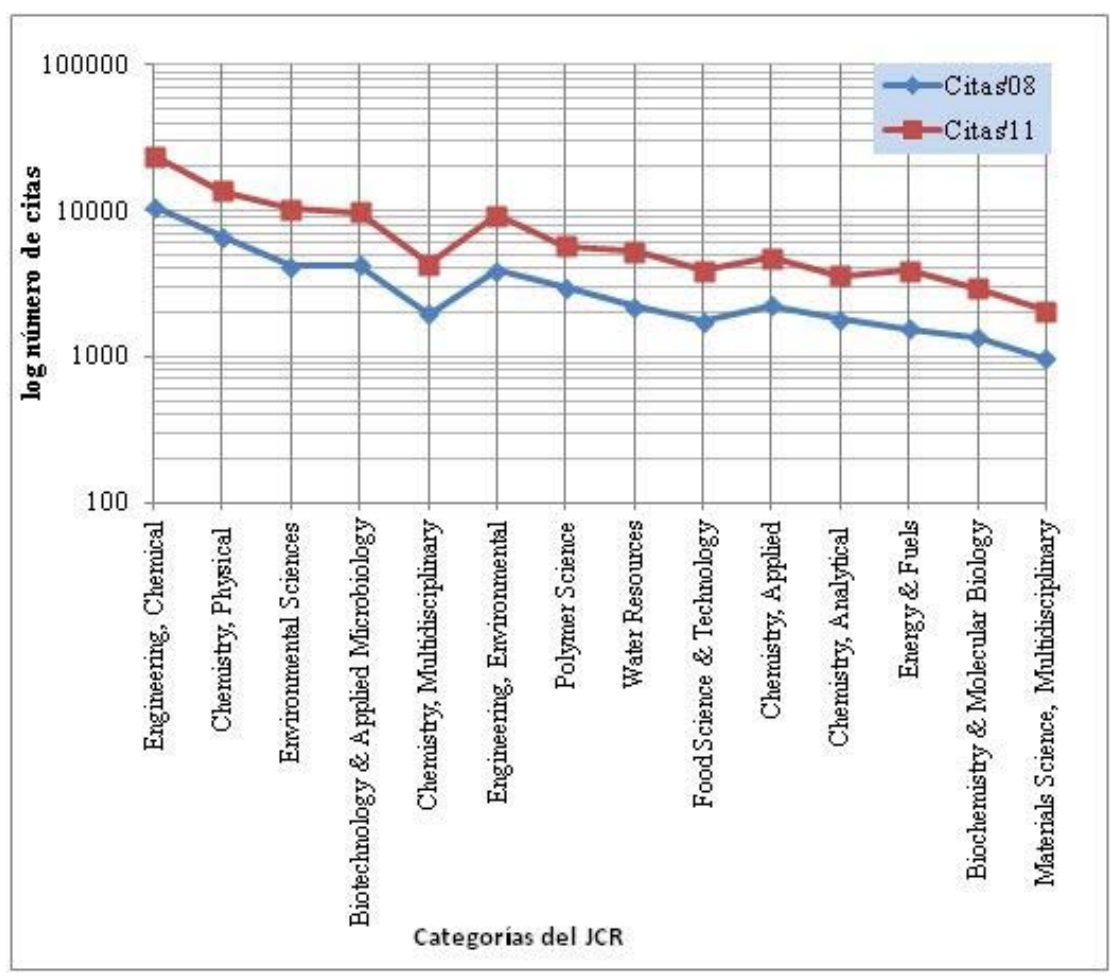


Figura 3. Mapa de visualización de similaridades de las 103 categorías del JCR analizadas

$$
\begin{aligned}
& \text { Agricuture, Dairy \& Animal sc } \\
& \text { Food Science \& Technology }
\end{aligned}
$$

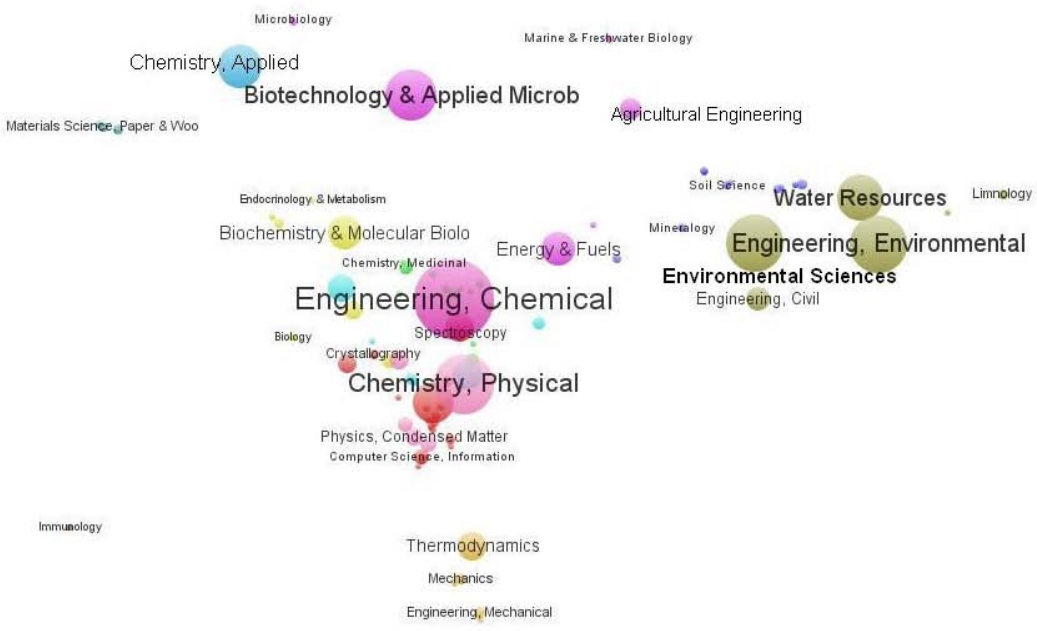

Las categorías situadas en el centro del mapa nos indican una fuerte coocurrencia con otras categorías, estando por tanto muy relacionadas. Las categorías más centrales son: "Engineering, Chemical", "Chemistry, Physical" son las categorías más relacionadas con el resto de áreas al mismo tiempo que son las que presentan una mayor coocurrencia; también la categoría "Biotechnology \& Applied Microbiology" destaca por una centralidad y coocurrencia bastante significativa.

Aquellas categorías situadas cerca indican que ocurren a menudo juntas, mientras que categorías situadas lejos unas de otras indican una escasa coocurrencia al mismo tiempo. Observando la fig. 3, la categoría "Chemical, Engineering" presenta una fuerte coocurrencia con áreas cercanas como: "Chemistry, Physical", "Energy \& Fuels", "Biochemistry \& Molecular Biology", "Chemistry, Organic" y "Chemistry, Analytical". Las categorías que se encuentran en el borde del mapa coocurren con un pequeño número de categorías, suelen pertenecer a campos aislados; tal es el caso de: "Agriculture, Dairy \& Animal Science", "Immunology" o "Engineering, Mechanical".

La fig. 4 muestra la densidad del mapa de coocurrencias de categorías. La densidad de un área en el mapa está determinada por el número de términos en el área y por la frecuencia con la que los términos ocurren. El tamaño de los términos y la densidad de las diferentes áreas indican su nivel de importancia. Cada área de investigación está representada por un punto en el mapa cuyo color estará entre el rojo y azul. El color del punto dependerá del número de categorías alrededor y de la intensidad de coocurrencia de éstas en el estudio. Un punto será más rojo cuando las categorías alrededor sean más nu- merosas y tengan una coocurrencia alta, mientras que tenderá a azul cuando el número de categorías alrededor sea pequeño con una coocurrencia mínima. Esta vista es muy útil para obtener una visión general de los aspectos más importantes del mapa (Van Eck y Waltman, 2010).

En el mapa de densidad se pueden observar dos áreas determinantes: "Engineering, Chemical" y "Chemistry, Physical". También es importante destacar el área de densidad de "Engineering, Environmental", muy interrelacionada con categorías como "Water Resources" y "Environmental Sciences". Otra área que merece ser mencionada por su centralidad y tamaño es "Biotechnology \& Applied Microbiology".

La tabla IV contiene las categorías con mayor frecuencia de ocurrencias, destacando: "Engineering, Chemical" (cluster 13), "Chemistry, Physical" (cluster 10), "Engineering, Environmental" (cluster 9), "Environmental Sciences" (cluster 9) y "Biotechnology \& Applied Microbiology" (cluster 5).

La tabla $\mathrm{V}$ muestra el número de categorías temáticas que engloba cada uno de los 13 clusters. El tamaño de un cluster está determinado por factores tales como: número de términos en el cluster, frecuencia de ocurrencia de los términos y la fuerza con la que los términos se relacionan entre sí. Clusters más centrales indican una alta relación con términos diferentes, mientras que clusters situados en el borde del mapa sugieren una menor relación con otras categorías. El cluster 13 integrado por la categoría "Engineering, Chemical" es el cluster más central y por tanto el que presenta una interdisciplinariedad mayor; mientras que el cluster no 11 (Allergy, Immunology Surgery y Transplantation) presenta una interrelación menor. 
Figura 4. Mapa de densidad de coocurrencias de las 103 categorías del JCR analizadas

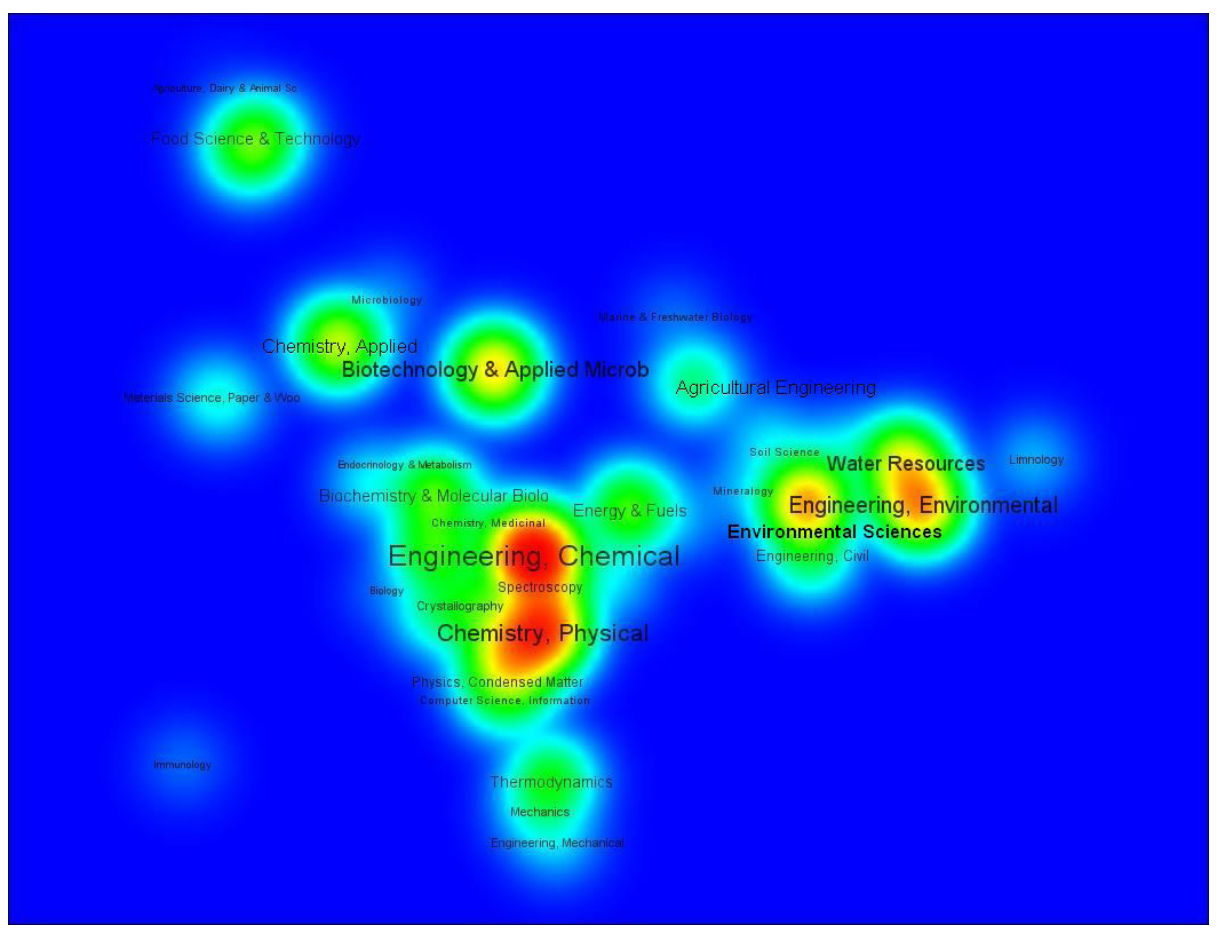

Tabla IV. Categorías con mayor frecuencia de ocurrencias

\begin{tabular}{|c|c|c|}
\hline Categoría & Cluster & Total link strength \\
\hline Engineering, Chemical & 13 & 3216 \\
\hline Chemistry, Physical & 10 & 1764 \\
\hline Engineering, Environmental & 9 & 1668 \\
\hline Environmental Sciences & 9 & 1618 \\
\hline Biotechnology \& Applied Microbiology & 5 & 1306 \\
\hline Water Resources & 9 & 1076 \\
\hline Chemistry, Applied & 7 & 940 \\
\hline Chemistry, Multidisciplinary & 1 & 864 \\
\hline Biochemistry \& Molecular Biology & 4 & 586 \\
\hline Energy \& Fuels & 5 & 580 \\
\hline Food Science \& Technology & 7 & 514 \\
\hline Materials Science, Multidisciplinary & 6 & 458 \\
\hline Chemistry, Analytical & 1 & 414 \\
\hline Thermodynamics & 8 & 412 \\
\hline Polymer Science & 6 & 380 \\
\hline
\end{tabular}

Tabla V. Número de categorías en cada cluster

\begin{tabular}{|c|c|c|c|c|c|c|c|c|c|c|c|c|c|}
\hline Cluster & 1 & 2 & 3 & 4 & 5 & 6 & 7 & 8 & 9 & 10 & 11 & 12 & 13 \\
\hline No de categorías & 22 & 13 & 12 & 10 & 8 & 7 & 6 & 6 & 6 & 5 & 4 & 3 & 1 \\
\hline
\end{tabular}




\section{DISCUSIÓN Y CONCLUSIONES}

Las 14 categorías del JCR seleccionadas en el estudio como las más productivas presentan una alta multiasignación. El 54\% de las revistas (324) y el $56 \%$ de los documentos estarían multiasignados, mientras que 274 revistas estarían asignadas a una sola categoría $(45,82 \%)$. Estos datos difieren de los del trabajo de Sanz y otros (2002), sobre la química puertorriqueña, en el que encuentran una interdisciplinariedad próxima al 30\%. Por categorías, estas varían desde el 47,5\% de "Polymer Science" hasta el $100 \%$ en el caso de "Engineering, Environmental" y de "Chemistry, Applied", contrastando estos datos, también, con los obtenidos por Morillo y otros (2001), en el área de la química, donde la interdisciplinariedad es más baja que la del presente trabajo; por ejemplo, "Chemistry, Applied", con 83,3\% o "Polymer Science", con $39,1 \%$. Zhang y otros (2010), en un estudio de todas las categorías del JCR, obtienen un $60 \%$ de revistas asignadas a una sola categoría, deduciéndose que las multiasignadas tendrán un porcentaje próximo al $40 \%$, un valor inferior a los obtenidos en el presente estudio.

De acuerdo con Hurd (1992), quien dice que la interdiscipinariedad es la demanda de ideas, conceptos, instrumentos, etc. de otras disciplinas, creemos que la IQ, requiere de otras muchas disciplinas como apoyo o complementarias. La IQ de hoy día se basa en tres grandes sectores: 1 ) Operaciones básicas y operaciones unitarias; 2) Ingeniería de la reacción química; 3) Ingeniería de procesos químicos-industriales. Para afrontar estos retos necesita de disciplinas como la termodinámica, la cinética química, fenómenos de transporte, control y dinámica de sistemas, economía y un número indeterminado de otras disciplinas más específicas como son: ciencias de los materiales, biotecnología, tecnología de los alimentos, electrotecnia, termotecnia, tecnología del medio ambiente, biotecnología, etc. Por todo ello la IQ es más interdisciplinar que la química, ya que esta última demanda de un número menor de disciplinas para acometer sus investigaciones.

Respecto a la colaboración de los autores (índices de coautoría), no se aprecian diferencias significativas entre los correspondientes a una sola categoría asignada o a multiasignadas, ni entre los distintos niveles de asignación (test de KruskalVallis; $\left.\chi^{2}=5 ; p=0,416\right)$. A estas mismas conclusiones se llega en el trabajo de Sanz y otros (2002). Tampoco se apreciaron diferencias entre los índices de coautoría de las 14 categorías del JCR seleccionadas como más productivas. Estos hallazgos indicarían que no existe relación entre la interdisciplinariedad y el número de autores de los trabajos científicos en IQ, si bien Qin y otros (1997) muestran diferencias significativas en el grado de interdisciplinariedad entre los diferentes niveles de colaboración y entre diferentes disciplinas y concluyen que la colaboración contribuye sig- nificativamente al grado de interdisciplinariedad en algunas disciplinas y no en otras.

Las citas recibidas por las distintas fuentes, recogidas en dos períodos de tiempo (2008 y 2011), se representaron por conjuntos de revistas multiasignadas a cada categoría del JCR, en cada uno de los dos períodos. Los resultados muestran una alta correlación $(r=0,998)$ entre citas'08 y citas' 11 . Esto indica que el comportamiento de la citación en un conjunto de fuentes multiasignadas a una categoría del JCR se mantiene, al menos en períodos cortos de tiempo.

Sin embargo, entre las citas recibidas por las revistas asignadas a una sola categoría y las recibidas por el conjunto de revistas multiasignadas, no hubo diferencias significativas $(t=1,872 ; \mathrm{p}=$ 0,135 , para citas'08 y $t=1,693 ; p=0,156$, para citas'11). Estos resultados significan que el hecho de que una revista esté asignada a una sola categoría o a varias, no influye en el número de citas que recibe, al menos en IQ.

Se ha estudiado la visibilidad de los documentos a través del porcentaje de revistas en el $1^{\text {er }}$ cuartil (publicaciones de 2006). Esta ha mostrado diferencias notables según la categoría, variando desde un $29 \%$ al $52 \%$. Los porcentajes calculados para los documentos publicados en esas mismas revistas arrojan valores superiores, a excepción de "Water Resources", con un $9,8 \%$. El resto varía entre el $45 \%$ de "Chemistry, Analytical" y el $90 \%$ de "Energy \& Fuels". Un comportamiento similar fue apreciado en los químicos puertorriqueños (Sanz y otros, 2002).

El estudio de las fuentes de las referencias de los artículos publicados por los ingenieros químicos universitarios españoles revelaron una similitud, respecto a la multiasignación a las categorías, con las fuentes de publicación. Aunque el estudio se llevó a cabo solo con referencias de 2006, las 13 primeras categorías son idénticas a las de las fuentes de publicación, si bien el orden difiere en algunos casos. La categoría de "Engineering, Chemical" ocupa el primer lugar, con 18 revistas $(20,7 \%)$ y 3.722 documentos $(23,94 \%)$, seguida de "Chemistry, Applied" con 10 revistas $(11,5 \%)$ y 1.704 documentos $(10,96 \%)$ y de "Biotechnology \& Applied Microbiology", con 9 revistas y 1.419 documentos. Existe una fuerte correlación entre fuentes y documentos $\left(R^{2}=0,96\right)$

Con respecto a la colaboración internacional, las 14 categorías seleccionadas superan el $76 \%$ de la colaboración internacional, siendo las tres categorías más productivas las que mayor porcentaje de colaboración presentan (un 34\% entre las tres). "Engineering, Chemical" alcanza casi el 17\%.

Los mapas muestran la relación entre los distintos grupos de categorías. Estas agrupaciones aparecen formadas por 13 clusters, que varían entre 22 categorías (cluster 1 ) hasta 1 categoría (Engineering, Chemical, cluster 13). 
Respecto a la estructura que presenta la IQ, destacan "Engineering, Chemical,", "Chemistry, Physical" y "Biotechnology \& Applied Microbiology" por su significativa centralidad y coocurrencia. En el borde del mapa, como campos aislados, se muestran las categorías de: "Agriculture, Dairy \& Animal Science", "Immunology" o "Engineering, Mechanical". El mapa de densidad, determinado por el número de términos en el área y por la frecuencia con que estos ocurren, indica el nivel de importancia de las categorías. En este sentido destacan "Engineering, Chemical" y "Chemistry, Physical". También es de destacar "Engineering, Environmental", por su interrelación con "Water Resources" y con "Environmental Sciences".

Aunque la metodología utilizada para analizar la interdisciplinariedad de un campo científico ha sido rica y variada, según la literatura consultada (a través de los documentos, las revistas, la autoría, las citas, etc.), creemos que el hecho de abordarlo desde las fuentes, donde se publican los documentos, fue una opción acertada, que cumplió con los objetivos marcados y que aportó resultados concluyentes. Las revistas como tales pueden ser tratadas desde un enfoque más objetivo que el que puede hacerse con los documentos o las propias citas. El hecho de observar la distribución de las fuentes entre las distintas categorías del JCR y la multiasignación de aquéllas en categorías resultó un método apropiado para medir la interdisciplinariedad $y$, también, para poder comparar los distintos campos científicos, independientemente de su especialización. Igual apreciación que para las fuentes se podría indicar para las bases de datos WoS de Thomson Reuters. Se trata de unas bases de datos apropiadas para este tipo de estudio, por su contenido, visualización, recuperación de la información, disposición de los datos, estructura de las categorías del JCR, internacionalidad, etc.

Los resultados apoyan la utilidad de este tipo de estudio para una mejor comprensión de la interdisciplinariedad en un área de conocimiento. En este caso se ha tratado un área muy dinámica de la química, como es la Ingeniería Química, de la que se han analizado las distintas categorías del JCR, a las que se asignan las revistas donde publican los ingenieros químicos. Se han estudiado una serie de indicadores con los que se han observado las diferencias entre las categorías, así como algunas limitaciones de esos indicadores.

\section{BIBLIOGRAFÍA}

Bordons, M.; Morillo, F.; Gómez, I. (2004). Analysis of cross-disciplinary research through bibliometric tools". En Moed, H. F.; Glänzel, W.; Schmoch, U. (editores.) Handbook of Quantitative Science and Technology Research. The Use of Publication and Patent Statistics in Studies on S\&T Systems, p. 437-456, Kluwer Academic Publishers.

Bordons, M.; Zulueta, M. A.; Romero, F.; Barrigón, S. (1999). Measuring interdisciplinary collaboration within a university: The effects of the Multidisciplinary Research Programme. Scientometrics, vol. 46 (3), 383-398. http://dx.doi.org/10.1007/BF02459599

Carayol, N.; Thuc Uyen, N. T. (2005). Why do academic scientists engage in interdisciplinary research? Research Evaluation, vol. 14 (1), 70-79. http:// dx.doi.org/10.3152/147154405781776355

Chang, Y. W.; Huang, M. H. (2012). A study of the evolution of interdisciplinarity in library and information science: using three bibliometric methods. Journal of the American Society for Information Science and Technology, vol. 63 (1), 22-33. http://dx.doi.org/10.1002/asi.21649

Cronin, B.; Pearson, S. (1990). The export of ideas from information science. Journal of Information Science, vol. 16, 381-391. http://dx.doi. org/10.1177/016555159001600606

Hargens, L. L. (1986). Migration patterns of U.S. Ph.D.S among disciplines and Specialties. Scientometrics, vol. 9 (3-4), 145-164. http://dx.doi.org/10.1007/ BF02017238

Hurd, J. M. (1992). The Future of University Science and Technology Libraries: Implications of increasing interdisciplinarity. College \& Research Libraries, vol. 13 (1): 17-32.

Izenman, A. J. (2008). Modern multivariate statistical techniques: Regression, classification and manifold learning. New York: Springer. http://dx.doi. org/10.1007/978-0-387-78189-1

Katz, S.; Hicks, D. (1995). The classification of interdisciplinary journals: a new approach. En Koening, M. E. D.; Bookstein, A. (Eds.) Proceedings of the Fifth Biennial Conference of the International Society for Scientometrics and Informetrics, $\mathrm{p}$. 245-254. Medford: Learned Information.

Lagar Barbosa, M. P. (2012). Análisis cuantitativo de la producción científica y técnica de la Ingeniería Química española. Tesis doctoral. Universidad de Extremadura, p. 1-493.

Le Pair, C. (1980). Switching between academic disciplines in universities in the Netherlands. Scientometrics, vol. 2 (3), 177-191. http://dx.doi. org/10.1007/BF02016696

Lu, K.; Wolfram, D. (2010). Geographic characteristics of the growth of informetrics literature 1987-2008. Journal of Informetrics, vol. 4 (4), 591-601. http:// dx.doi.org/10.1016/j.joi.2010.06.008

Morillo, F., Bordons, M., Gómez, I. (2001). An approach to interdisciplinarity through bibliometric indicators. Scientometrics, vol. 51 (1), 203-222. http://dx.doi. org/10.1023/A: 1010529114941

Morillo, F.; Bordons, M.; Gómez, I. (2003). Interdisciplinarity in science: a tentative typology of disciplines and research areas. Journal of the American Society for Information Science, vol. 54 (13), 12371249. http://dx.doi.org/10.1002/asi.10326

Pino-Díaz, J.; Jiménez-Contreras, E.; Ruíz-Baños, R.; y Bailón-Moreno, R. (2011). Evaluación de redes tecnocientíficas: la red española sobre Áreas Protegidas, según la Web of Science. Revista Española de Documentación Científica, vol. 34(3), 331-333.

Porter, A. L.; Chubin, D. L. (1985). An indicator of crossdisciplinary research. Scientometrics, vol. 8 (3-4), 161-176. http://dx.doi.org/10.1007/BF02016934 
Qin, J.; Lancaster, F. W.; Allen. B. (1997). Types and Levels of Collaboration in Interdisciplinary Research in the Sciences. Journal of the American Society for Information Science, vol. 48 (10), 893-916. http://dx.doi.org/10.1002/(SICI)10974571(199710)48:10<893: :AID-ASI5>3.0.CO;2-X

Rinia, E. J.; Van Leeuwen, T. N.; Bruins, E. E. W.; Van Vuren, H. G.; Van Raan, A. F. J. (2002). Measuring knowledge transfer between fields of science. Scientometrics, vol. 54 (3), 347-362. http://dx.doi. org/10.1023/A:1016078331752

Sanz, E.; Martín, C.; Maura, M.; Rodríguez, B.; García-Zorita, C.; Lascuraín, M. L. (2002). Análisis de la interdisciplinariedad de los investigadores puertorriqueños en ciencias químicas durante el período 1992-1999. Revista Española de Documentación Científica, vol. 25 (4), 421-432.

Schoepflin, U.; Glänzel, W. (2001). Two decades of "Scientometrics": An interdisciplinary field represented by its leading journal. Scientometrics, vol. 50 (2), 301-312. http://dx.doi. org/10.1023/A:1010577824449

Tijssen, R. J. W. (1992). A quantitative assessment of interdisciplinarity structures in science and technology: co-classification analysis of energy research. Research Policy, vol. 22 (1), 27-44. http://dx.doi.org/10.1016/0048-7333(92)90025-Y
Tijssen, R. J. W. (2010). Discarding the 'basic science/ applied science' dichotomy: A knowledge utilization triangle classification system of research journals. Journal of the American Society for Information Science and Technology, vol. 61 (9), 1842-1852. http://dx.doi.org/10.1002/asi.21366

Urata, H. (1990). Information flows among academic disciplines in Japan. Scientometrics, vol. 18 (3-4), 309-319. http://dx.doi.org/10.1007/BF02017767

Van Eck, N. J.; Waltman, L. (2007). VOS: a new method for visualizing similarities between objects. En: Lenz, H.-J.; Decker, R. (editores). Advances in Data Analysis: Proceedings of the 30th Annual Conference of the German Classification Society, p. 299-306. Berlín, New York: Springer.

Van Eck, N.J.; Waltman, L. (2010). Software survey: VOSviewer, a computer program for bibliometric mapping. Scientometrics, vol. 84 (2), 523-538. http://dx.doi.org/10.1007/s11192-009-0146-3 PMid:20585380 PMCid:PMC2883932

Zhang, L., Liu, X., Janssems, F., Liang, L., Glänzel, W. (2010). Subject clustering analysis based on ISI category classification. Journal of Informetrics, vol. 4 (2), 185-193. http://dx.doi.org/10.1016/j. joi.2009.11.005 